\title{
Catheter-related atrial thrombosis in a patient with recent cerebral haemorrhage: to treat or not to treat?
}

\author{
Martino F. Pengo • Paola Caielli • Fabio Ragazzo • \\ Gian Paolo Rossi
}

Received: 19 July 2010/Accepted: 10 November 2010/Published online: 9 December 2010

(c) SIMI 2010

\section{Case report}

We report on the case of a 43-year-old Indian man, brought to the emergency department (ED) because of traumatic brain injury due to a motorcycle crash. In the $\mathrm{ED}$, the patient was awake, and haemodynamically stable. The neurological examination did not reveal any abnormality. All standard blood tests were normal. A brain computed tomography (CT scan) showed blood leakage on cortical insular and frontal furrows, temporo-parietal subdural haematoma, and a left occipital skull fracture. As the neurosurgeon suggested a conservative approach, the patient was transferred first to an ICU, then to our unit. Over the next few days, he became pyretic, and therefore the central venous catheter was removed. The catheter's tip cultures eventually were positive for Staphylococcus epidermidis. A transthoracic echocardiogram (TTE) showed a right atrial mass (Fig. 1), which was determined to be an atrial thrombus on a transesophageal echocardiogram (TEE) and cardiac magnetic resonance imaging (MRI). Cautious anticoagulant therapy with low-molecular weight heparin was started, along with close clinical and radiological observation to detect any subtle haemorrhagic complications. After 20 days of effective anticoagulation, a TTE showed no evidence for atrial thrombi (Fig. 1).

M. F. Pengo ( $₫)$ · P. Caielli · F. Ragazzo - G. P. Rossi

Department of Clinical and Experimental Medicine (DMCS),

Clinica Medica 4, University of Padova, Padua, Italy

e-mail: martino.pengo@unipd.it

\section{Discussion}

Catheter-related thrombosis (CRT) occurs in $14-36 \%$ of patients within 1-2 years of long-term central venous catheter placement [1] and is a common complication also for intensive care unit patients with an average reported rate of $19.3 \%$ [2]. A frequent consequence of CRT is thromboembolism, which increases associated morbidity and mortality [3]. Unfortunately CRT is under-diagnosed because of difficulty in thinking of the problem and in confirmation of the diagnosis since often CRT develops in a totally asymptomatic fashion. Moreover, there also is a lack of reliable data on the risk factors of CRT: a few studies suggest that besides the patient's clinical features [2], the catheter material, the position of the catheter tip, concurrent infections, previous catheterization, and other factors may influence the risk of CRT [1].

The choice of the optimal treatment is also challenging, since there are no guidelines to suggest the best approach to CRT, especially when it is complicated with right heart thrombosis.

This is likely because by far the vast majority of right heart thromboemboli entail embolized material from deep venous thromboses temporarily lodged in the right atrium or ventricle, which are usually referred to as emboli in transit. In fact, nearly all patients with right heart thromboembolism have coexisting massive bilateral pulmonary emboli [4]. Based on a systematic review of the literature Rose et al. [4] conclude that the most effective therapy for patients with right heart thromboemboli remains unknown. This is very worrying since the presence of a right heart thromboembolus, particularly complicating a pulmonary thromboembolism, carries a poor prognosis. Chartier et al. [5] report a mortality rate of $45 \%$ in the most recent series of 38 consecutive patients with right heart thromboembolism. 
Fig. 1 A transthoracic echocardiogram (pre) showing a mass in the right atrium. After 20 days of effective anticoagulation a transthoracic echocardiogram (post) showed no evidence for atrial thrombi

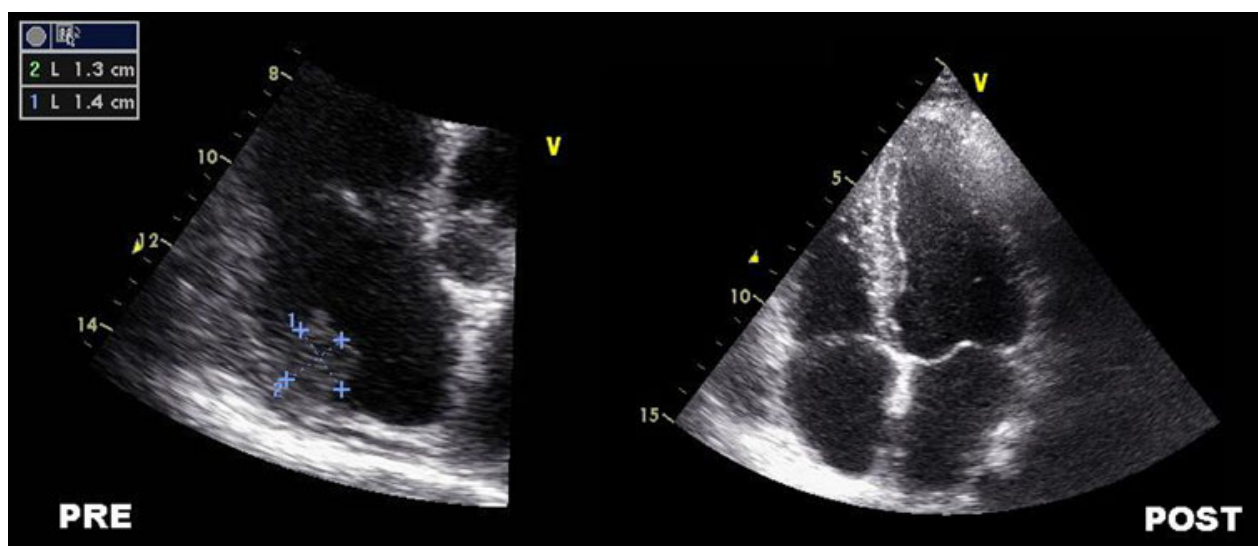

All these deaths occurred within the first $24 \mathrm{~h}$ of hospitalization, underscoring the need for a swift diagnosis and treatment of right heart thromboembolism. Existing published reports differ in their recommendations for treatment by advocating surgical removal, the administration of thrombolytic agents or anticoagulation therapy with heparin. [5].

An analysis of 119 published patients with right heart thromboembolism reports similar mortality rates for surgery, thrombolysis, and heparin anticoagulation therapy $(38,38$, and $30 \%$, respectively), which, oddly enough, led the authors to conclude that heparin therapy was the treatment of choice. At variance, a recent survey of all patients with right heart thromboembolism that have been reported in the English language literature shows that thrombolytic therapy is associated with a reduction in mortality compared to anticoagulation or surgical therapy. [4].

In the present case a CRT, likely induced by a concomitant infection, was complicated with right atrial thrombus and required immediate treatment to prevent fatal pulmonary embolism. Notwithstanding a coexisting relative contraindication to anticoagulation (recent cerebral haemorrhage), after in-house consultation, it was decided to treat the patient with low-molecular weight heparin with a close clinical and radiological follow up. This treatment resulted in the dissolution of the atrial thrombus without any haemorrhagic complication.

Hence, in the lack of strong recommendations concerning the optimal treatment of catheter-related atrial thrombosis and atrial thrombi, particularly when contraindication to anticoagulation or thrombolytic therapy exist, physicians are left with a difficult choice. It is unlikely that there will ever be enough cases to allow undertaking a prospective randomized trial on this issue in order to achieve a better guidance for the treatment of this condition. In our view even when contraindications to reperfusion therapy are present, the prospect of a bleeding complication is worth enduring to try to obtain a better outcome because otherwise the outcome will be awful.

Conflict of interests None.

\section{References}

1. Baskin JL, Pui CH, Reiss U, Wilimas JA, Metzger ML, Ribeiro RC, Howard SC (2009) Management of occlusion and thrombosis associated with long term indwelling central venous catheters. Lancet Jul 11; 374(9684):159-69. doi:10.1016/S0140-6736(09) 60220-8

2. Klerk CP, Smorenburg SM, Büller HR (2003) Thrombosis prophylaxis in patient populations with a central venous catheter. Arch Intern Med 163:1913-1921

3. Frizzelli R, Tortelli O, Di Comite V, Ghirardi R, Pinzi C, Scarduelli C (2008) Deep venous thrombosis of the neck and pulmonary embolism in patients with a central venous catheter admitted to cardiac rehabilitation after cardiac surgery: a prospective study of 815 patients. Intern Emerg Med 3:325-330. doi: 10.1007/s11739-008-0142-2

4. Rose PS, PunjabiNM, Pearse DB (2002) Treatment of right heart thromboemboli. Chest 121:806-814. doi:10.1378/chest.121.3.806

5. Chartier L, Béra J, Delomez M, Asseman P, Beregi JP, Bauchart JJ, Warembourg H, Théry C (1999) Free-floating thrombi in the right heart: diagnosis, management, and prognostic indexes in 38 consecutive patients. Circulation 99:2779-2783 